\title{
Management of Wireless Communication Systems Using Artificial Intelligence-Based Software Defined Radio
}

\author{
https://doi.org/10.3991/ijim.v14i13.14211
}

\author{
Faiq A. Mohammed Bargarai ${ }^{(凶)}$, Adnan Mohsin Abdulazeez \\ Duhok Polytechnic University, Duhok, Iraq \\ faiq.ahmededpu. edu.krd \\ Volkan Müjdat Tiryaki \\ Siirt University, Siirt, Turkey \\ Diyar Qader Zeebaree \\ Research Center of Duhok Polytechnic University, Duhok, Iraq
}

\begin{abstract}
The wireless communication system was investigated by novel methods, which produce an optimized data link, especially the software-based methods. Software-Defined Radio (SDR) is a common method for developing and implementing wireless communication protocols. In this paper, SDR and artificial intelligence (AI) are used to design a self-management communication system with variable node locations. Three affected parameters for the wireless signal are considered: channel frequency, bandwidth, and modulation type. On one hand, SDR collects and analyzes the signal components while on the other hand, AI processes the situation in real-time sequence after detecting unwanted data during the monitoring stage. The decision was integrated into the system by AI with respect to the instantaneous data read then passed to the communication nodes to take its correct location. The connectivity ratio and coverage area are optimized nearly double by the proposed method, which means the variable node location, according to the peak time, increases the attached subscriber by a while ratio.
\end{abstract}

Keywords - SDR, artificial intelligence, connectivity, coverage area.

\section{Introduction}

Nowadays, internet communication becomes a major part of infrastructure. Based on the internet most of the applications of infrastructure systems can be operated [1]. The interest in the research of the use of artificial intelligence (AI) and software-defined ratio (SDR) has gained momentum in the management of networks and communication systems in the last couple of years. The reason for extensive research in SDR concepts is to transfer the radio engineering problem mainly to the software domain rather than the hardware domain. However, the idea of coming up with new communication devices has introduced the ability to adapt a self-regulated fashion in terms of work, whether there is proper modulation selection for a signal transmitted and demodulation 
for the signal received or not. The appropriate distribution of nodes in a given area noted that there is more attention that is given to the wireless network research $[2,3]$ as shown in figure 1 .

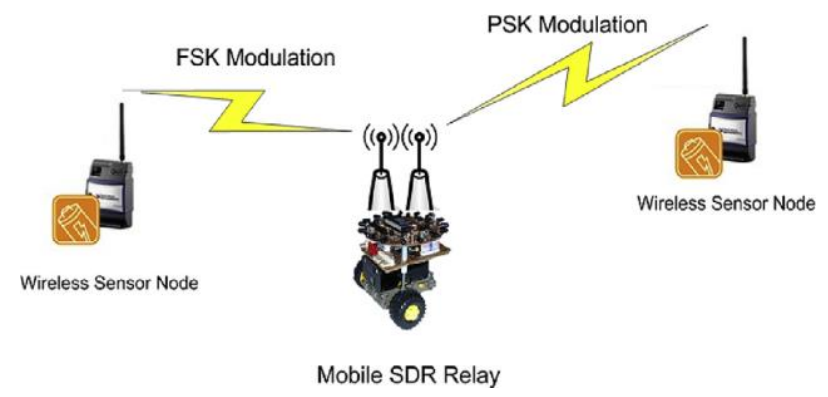

Fig. 1. Mobile relay devices supporting SDR capabilities

The introduction of powerful hardware platforms and software reconfiguration marks a new chapter on communication advancement. The capabilities of SDR are the only hopes for the anticipated future wireless devices, which can be enhanced through coupling controlled mobility $[4,5]$. The scope of networks as well as communication management attracts and impacts the users and system management to interact with various network communication tasks while incorporating SDR and the ANN [6, 7].

In this research, the coverage and connectivity on the application of SDR and ANN in network management and communication systems are what has been focused on to improve the QoS[8].

The radio devices of the traditional hardware era make cross-functionality harder and can only be modified through physical intervention. Such a constraint makes traditional communication have higher production costs and also lacks the flexibility to cope with the multiple waveform standards[9].

The SDR is expected to be utilized in the next generation of wireless networks and to introduce new architectural frameworks for wireless software[10].

A majority of the existing wireless networks are hardware-based and use architectural designs that are closed or inflexible. They make scalability hard and it is difficult to add new hardware devices in such a wireless network. When the communication system has an inflexible hardware-based architecture, it takes a 10-year cycle to deploy and standardize it with a new generation of wireless networks. The wireless network research community has been looking for ideas that will assist them in designing communication devices that will function in a self-organized manner and adapt to the changes that are experienced in the working environment[11].

For instance, the $(5 \mathrm{G})$ cellular network requirements for the ultra-high capacity is 1000 -fold capacity/km2 different from the long-term evolution (LTE). This fundamental transformation of the system needs time and also a significant cost that affects the commercial networks, prompting the researchers in thinking to select a more flexible network system as SDR[12].

The concept of designing new communication devices that can confirm their operational roles in a self-organized way to cope with rapid changes in the work environment 
has gained considerable attention from the wireless research community[13]. The availability of new general-purpose and powerful hardware platforms can be dynamically reconfigured through a program[14]. It paves the way for new research trends where complex communication scenarios can be deployed[15].

The new direction of communication systems and the advantages offered by SDR models were taken into account as envisioned in $[12,16]$. Researchers thought about designing a self-propagation strategy on adaptation communications network where many wireless devices are distributed in a specific area and can perform a common task according to the particular network requirements in terms of coverage or connectivity. The management system advantages of SDR is that it is the most suitable modulation scheme that can be achieved effectively. A single SDR system can be implemented to many radio systems (or radars) with different devices by only changing the software[17, 18].

Another aspect of the present research is to investigate the advantages of system management by software, which in turn leads to a high-quality result in the communication system by using SDR as the multi-channel. The switching modulation scheme can increase the rate of baud or reduce it to the maximum use of frequency channel capacity[19]. Furthermore, the general performance of the system can be improved in terms of connectivity which belongs to the SDR capabilities supported by a wireless node as well as a controlled mobility function, where the requirements for the restricted QoS connectivity are more stringent with regard to the maximum coverage $[4,13,20]$.

\section{Related Work}

In this research, two topics are focused on with regard to communication systems management. The first is concerned with SDR capabilities and the second one is concerned with the nodes' mobility capabilities for different communication scenarios. The system analysis was performed in this study and objectives included combination in a synergic way the (AI, SDR) capabilities and control mobility nodes. In order to have the management of communications systems with modern technology, we have designed an algorithm to select the best location for nodes and the most suitable modulation/demodulation scheme for each node distributed in a specific area. According to these observations, we first mention some of the contributions of positioning techniques to the wireless nodes that have attracted considerable interest in research as well as a role for SDR in managing the system to improve the QoS, which have become increasingly important in recent years[3].

One of the contributions that led to the thought of resorting to the design of a mobile communications system and the use of SDRs is because it allows interoperability with other network devices, the wireless communication systems, and military industry, among others and, because of its abilities to change protocols in real-time[21].

The increase in terrorism, border incidents, natural disasters and other emergencies in the world have contributed to the need for wireless communications that use SDR. Critical situations require a temporary network in locations with no operational wireless 
infrastructure due to natural disasters, breakdowns, fires, tsunamis or power outages. These emergencies cause the need for information sharing among institutions[22].

The conventional hardware-based wireless network communication is limited to support one type of network. Conventional communication networks have problems like frequency bands, use of base stations, protocols and waveforms. It obstructs communication and hence emergencies may be difficult when there is interference in communication. SDR eliminates communication challenges in times of emergencies as shown in figure 2[23].

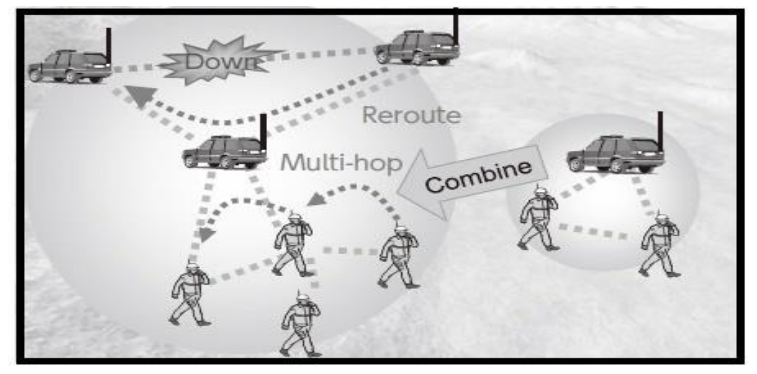

Fig. 2. Ad-hoc network example

On the other hand, the idea of using a modern system instead of traditional systems, which is the software system, instead of the hardware to manage the system and the reason for extensive research in SDR concepts is to transfer the radio engineering problem mainly to the software domain rather than the hardware domain[24, 25].

Through my work in one of the telecommunications companies, more than ten years I had worked in the section of control and towers and control signals and frequencies, which made me think of the search for a flexible technology to manage the system of the company, as there are a number of disadvantages that can be improved. This includes how to get rid of the harmonics noise and get the best signal, and also when needed in the development of the system of the company as the cost is high as well as time to change the system from $2.4 \mathrm{G}$ to $5 \mathrm{G}$, that is to change all the devices that belong to the company as well as the users, and this is what we want to get rid of. It will be simple to use the software instead of hardware because the software just needs to be updated which is the main purpose of this search[26].

The proposed method in this research enables the processing of the functions of the signal, in terms of architecture, the DAC/ADC usually brought close to an antenna as well as the processor, whereby the distance is made convenient enough for signal processing[27]. 


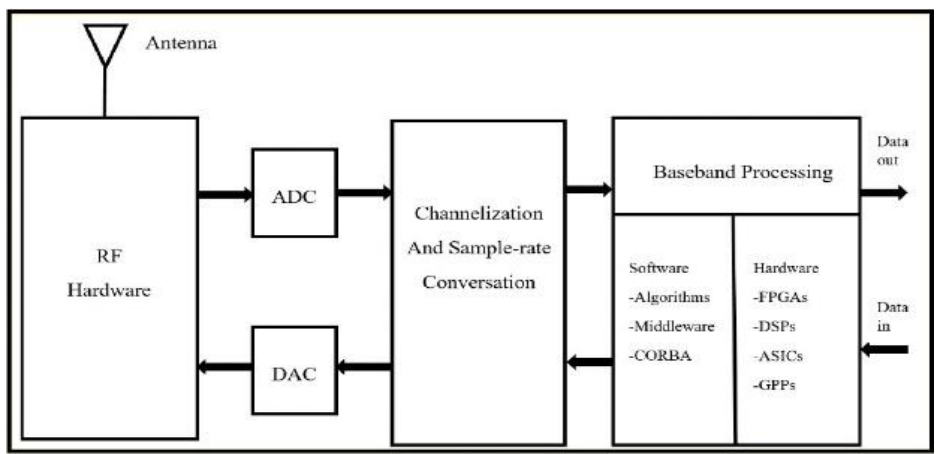

Fig. 3. SDR transceiver and receiver [28]

The other capabilities shared by [29] include the ones studied under the cognitive radio. Researchers have focused on SDR to implement structures on reconfigurable FPGAs, as well as signal processing using software instead of hardware[30].Some of the capabilities include a general-purpose processing capability that involves the DSP and FPGA processing resources. The resources never get optimized for the noted protocol applications. The same applies to AI applications. The SDR performance has the resources programmed in terms of the high-level languages.

Secondly, the cryptographic capabilities are seen in the military radios for security functions. They have an assurance system dedicated to the hardware. The programmable cryptographic processors were normally available to perform the security functions[20]. SDR technology ensures that communication is inexpensive and efficient. It allows multi-functional and multi-mode wireless devices to be incorporated into a network through software upgrades[31].

\subsection{The application of SDR}

SDR has been advantageous to most of the wireless communication systems. First, it plays a frontal role in terms of reducing manufacturing costs and development time. The application of the identical hardware platform for most of the terminals and protocols has the possibility of reducing the development costs and time to market by using SDR [32]. The second advantage is that SDR allows most of the service providers to upgrade the infrastructure with no additional costs and the wireless protocols can be improved to provide better services[33].Some typical examples include the W-CDMA protocol and the high-speed downlink packet access (HSDPA) channel. Also, the SDR avails seamless wireless connections to most of the end-users. These services form the key features of the $4 \mathrm{G}$ communication system[34].

\subsection{The impact of SDR in network management and communication system}

SDR technology has been applied in communication and network systems. According to the study done by [35] on the advancement noted in most of the cognitive radio networks, most of the devices are operating by the SDR principles. 
New technologies, as well as new algorithms, have enhanced the accuracy and efficiency of the wireless communication systems. While reviewing the impact of SDR on communication and network systems, it is essential to look at the air interface where the $(2 \mathrm{G})$ cellular systems seem to have narrowed down to the CDMA and TDMA techniques. However, modification of these systems would have enhanced the capabilities based on the conditions and the characteristics that are affected in case of any changes[36, 37].

The advancement has seen changes in data rates with subsequent generations enjoying better usage of improved systems. Under the SDR technology, the next generation will have hopes of a faster and accurate method that runs on the gigabyte interface. Another area of focus while fostering the impact of SDR technology, is the wireless technologies[20]. The progress and evolution of wireless technologies can attribute to the desire for a system with advanced reliability as well as availability. To some extent, the argument of management through radio access networks especially in densely populated areas can be justified[38].

The research covered by [7] takes an extra milestone in covering the integration of SDR with other technologies to impact the management process of the communication and network system. The coverage on the software-defined network (SDN) and the scope of the mobile network is essentially a new area of interest with regards to establishing a better and stronger management scheme. It is noticeable that every system has its own strengths and weaknesses, which is also a phenomenon witnessed in human beings.

In the network and communication world, the SDR system has been at the center of scheduling the channel priority while the SDN system is essentially engaged in monitoring and distributing traffic. Both SDN and SDR have their specific elements, architecture, and connection which are relevant to the central management. The recent development of SDR, however, sends a strong message of efficient decisions with more centralized systems coming in play[39].

In 2011 , the conventional technique that was in use included the $4 \mathrm{G}$, which is slightly different from the $5 \mathrm{G}$. It has compelled some researchers to set more preference on the service-oriented cognitive networks compared to the IP multimedia subsystems[38].

The proposed system sets more preference for radio channel scheduling while obtaining the most effective service experience. Based on the coverage by[7], the SDR layer has devices that can suit the expected properties of future mobile phones. It is because of some features that give it an upper hand compared to other systems. The administration component for this layer determines the frequency band and takes measures while eliminating any incident that can cause interference. On the other hand, the cross-layer controller only knows the request made for the frequency spectrum usage, which means that it can also determine the authorization of the device[40].

\subsection{Modulation schemes in SDR}

The analysis of the fixed node and support was given to SDR by[3]. The SDR capabilities were reviewed before common modulations that have appeared prior to including the MFSK, MQAM, and MPSK, which also take different symbols. The analysis of 
the mobile nodes can recommend any of the schemes. Over a third of the nodes might work in favor of PSK, QAM, and FSK. Such systems like the MFSK are usually called the M-ray signaling system and have the M-tones noted with the transmitter as one of the tones is selected for transmission[41]. However, the performance, as well as the characteristics of the system, is primarily determined through the modulation scheme chosen in the beginning.

\subsection{Artificial Neural Networks (ANNs)}

AI was introduced in 1943 when McCulloch and Walter Pitts wrote a document on the working of the neurons. The subsequent modeling of the electrical circuits brought out the picture of the neural network that reflected the neurons in the brain. The concept of neurons was later reinforced by Donald Hebb who noted the neural pathways. The advancement of computers in the 1950s made the beginning of the modeled rudiments possible, further attracting different theories concerning human thought[42].

Nathanial Rochester went ahead and simulated the ANNs with the first attempt being unsuccessful. The subsequent attempts came out more successful. More researchers came on board to contribute to AI. Some of them are Frank Rosenblatt, Bernard Widrow, Marcian Hoff, and John Hopfield among others. AI and GA were simply invented as a copy of the biological metaphor. The entire ANN mostly symbolized the human brain, which emanated into a computing model that constituted small interconnected units. In simple terms, AI is a mere computing system believed to carry out activities in a more defined connection system in the absence of the programming instructions. The network is typically trained to reflect samples of signals seen or observed in the human brain[43].

The expansion of computer networks and communication systems focused on the role of artificial intelligence in the management of systems from many aspects, including control and protecting data from penetration[44].

\subsection{The impact of ANN in the management of a wireless communication system}

ANNs were used in the management of the network and communication systems. According to[45], some of the intelligent tools under ANNs have made a significant impact in terms of solving the telecommunication problems. The limited bandwidth is largely restricted to the enormous growth of the well-managed telecommunications systems. Both the receiver and the transmitter can impact the transmission channel. In most cases, managing the communication and network system involves eliminating any form of disturbances. ANN stands out as an intelligent structure that can compensate for the interference. Therefore, the networks are good at ensuring that the system has effective control.

There has been significant research on Antenna Array Signal Processing, which mainly narrows down to direction-of-arrival DOA estimation as well as beamforming. ANNs have further received attention in their contribution towards CDMA, which has been convenient in handling the inherent channel noise. 
The impact of ANN on the network and communication system is also captured in the research done by [45]. ANNs have been a solution to most systems such as alarms. They have played a key role in terms of maintaining the service model where the service topology is involved.

The networks also have a stake in maintaining the impact rules as far as extensive programming is concerned. It is because AI is essentially an algorithm served with the ability of advanced pattern recognition. This advantage has made it more applicable in the alarm-priority patterns. Besides, the extensive study of machine learning has been useful in terms of impacting the emerging applications attached to communication technology that is witnessed in smart cities. The same sense applies to the emergence of the Internet of Things. The impact of ANN on the network and communication system is also recorded in the research done[45].

According to the research conducted by[46], the utilization of modern learning algorithms is an added milestone in communication as far as privacy matters are concerned. These machine learning methods have a big impact on the security section of the communication platform. The use of autoencoders provides the state-of-art approach towards enhancing some components such as video encoding and bit allocation.

Recently, artificial intelligence has gained an interesting interest for its direct role in the field of industry, medicine and many other fields[47].

\section{$3 \quad$ Materials and Methods}

In this part, a clear explanation of the system is theoretically presented throughout describing all the used tools and the proposed software and hardware aspects.

Through this work, SDR was used to manage and monitor the radio signal and update its parameters according to the environment.

\subsection{The system structure}

The flowchart of the system shown in figure 4, it is a step by step work, simulated by drawing the process for the overall proposed method. The SDR components are fixed at the first step then ANN and GA took place after the signal was recorded. The step condition was determined by the degree of the signal processed by RTL-SDR. AI controls the behavior of each node to obtain results, which in turn controls the direction of node movement as well as the role (of GA for TSP) in distributing nodes to select the shortest and best path for movement in a specific area to improve QoS in terms of coverage and connectivity. 


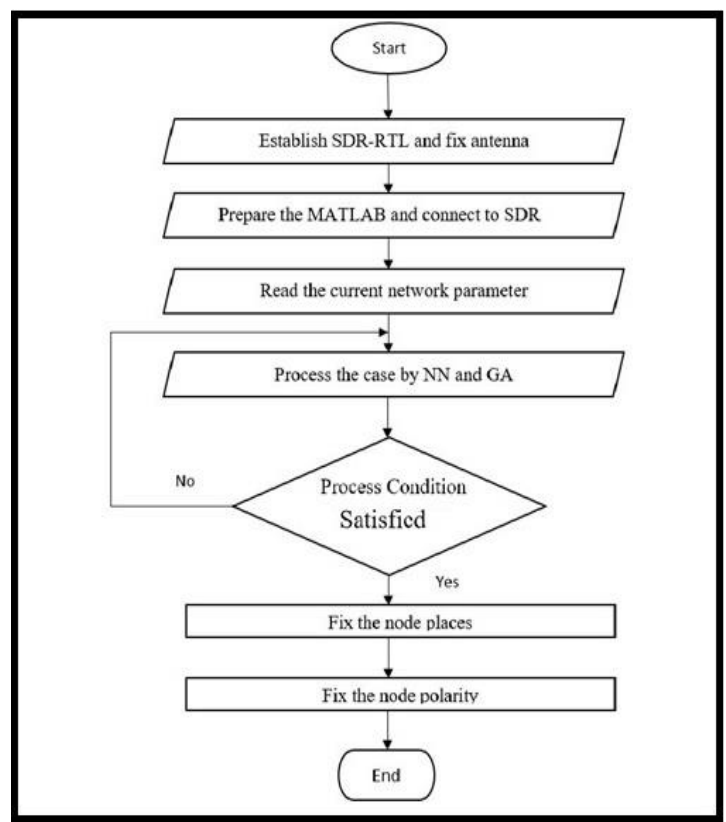

Fig. 4. SDR and ANN/GA Operation in System

\subsection{The SDR-RTL receiver}

The RTL-SDR radio receiver is a Realtek (RTL 2832U) chip which receives frequencies from $24 \mathrm{MHz}$ to $1.766 \mathrm{GHz}$, and bandwidth of $3.2 \mathrm{MHz}$ and has an 8-bit ADC resolution. It is a USB dongle thumb drive radio receiver used in different implementations such as FM radio, UHF TV broadcasting, 4G, LTE, and GPS systems. The RTLSDR is used to receive signals after processing and analyzing it according to the strength of the signal. This decision is made by the node and either remain fixed or moves in a certain direction.

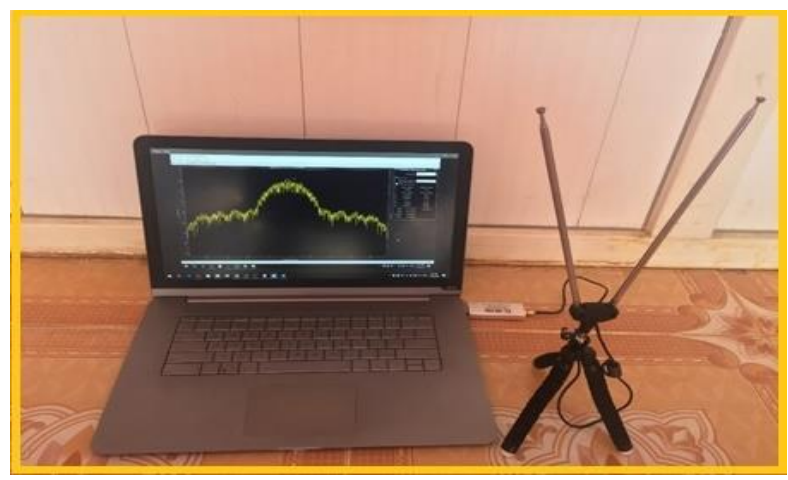

Fig. 5. Receiving signals by RTL-SDR 


\subsection{RTL-SDR receive FM signals}

The receiver portion of the system focuses on providing further details on the received signal and their processing. This is from the receiver point of RTL-SDR when the signal is received passes through several stages to be processed and obtains a pure signal, then this RF signal is sampled and demodulated, then decoded.

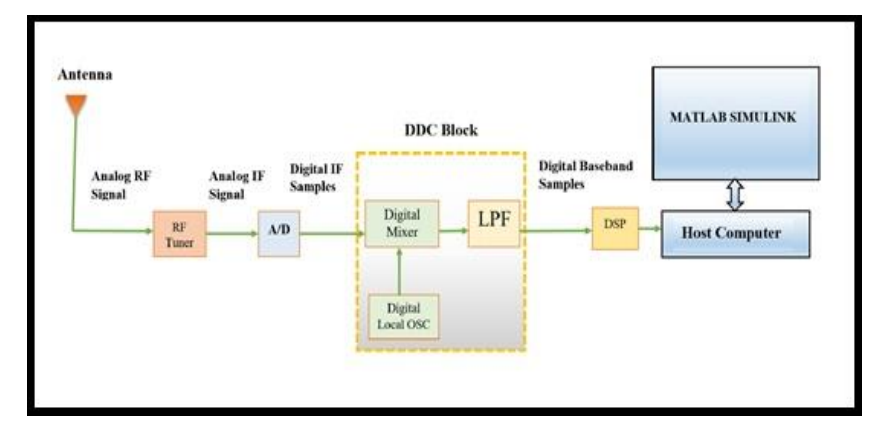

Fig. 6. SDR-Receiver architecture

Figure 7 is a block for RTL-SDR that contains an ADC and DSP in which the signal is demodulated together with all other operations needed to accomplish this, like filtering, decimation, amplification among others.

\subsection{The mathematical model}

Total Harmonic Distortion (THD) degradation means pure signal emission without causing interference to other electronic devices.

The problem of distorted radio transmission is very important in the context of spectrum sharing and spectrum sensing. The relationship between SINAD, SNR, and THD is given below. RTL-SDR reduces harmonic distortion to obtain a pure signal [46].

$$
\begin{gathered}
S N R=20 \log \left(\frac{S}{N}\right) \\
T H D=20 \log \left(\frac{S}{D}\right) \\
\text { SINAD }=20 \log \left(\frac{S}{N+D}\right) \\
\text { SINAD }=\frac{P_{\text {signal }}+P_{\text {noise }}+P_{\text {distortion }}}{P_{\text {noise }}+P_{\text {distortion }}}
\end{gathered}
$$

THD is measured and processed in this work by SDR and nearly six harmonic signals are detected with different amplitudes. The RTL-SDR took place of the complex hardware devices that are used to eliminate the effect of harmonics to the fundamental signal $[24,48]$. 


\subsection{The system management by SDR and ANN in a synergic way}

An integrated system was built and managed by ANN and SDR technology and the system has been improved by the GA for TSP. ANN was used to find the strength of each individual in the group, which in turn led to a sub-optimal solution to the problem. Information that flows through the network affects the weight coefficients of ANN in a sense based on information of input and output layers, as shown in figure 8 . The type of ANN used in this paper is the backpropagation ANN.

It is the most widely used ANN architecture. Many applications can be modeled using a backpropagation network. Backpropagation ANN has been successfully tested in this research and obtained a very high performance although the network does not contain links to the feedback, the errors are backward spread during the training process. The errors in the output layer determine error measurements in the hidden layer, which are used as a bias to modify the weights of the connection between the input layer and the hidden layer. Each repeated processing involves the adjustment of at least two sets of weights between a pair of layers and the rotation of the outputs. This treatment continues until the errors fall below the permitted threshold level. The ANN model used in this study shown in figure 7 consists of two hidden layers in addition to input and output layers.

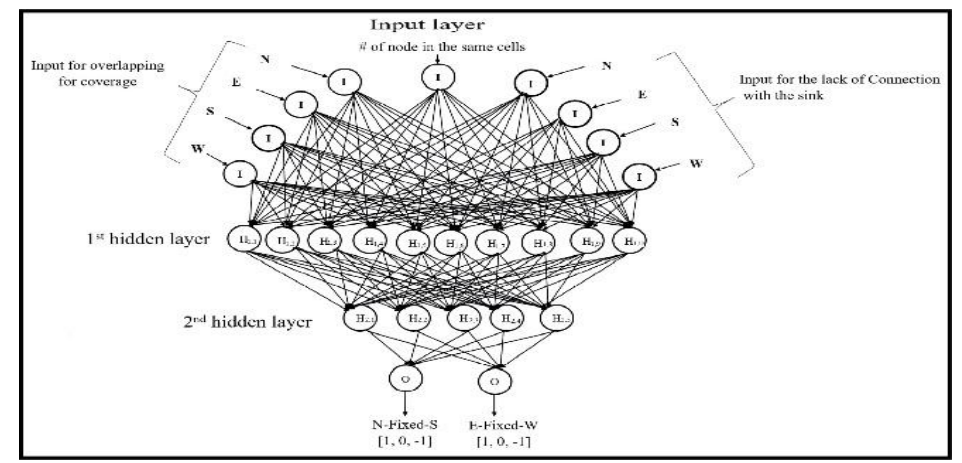

Fig. 7. Backpropagation ANN architecture of one node

For each node the output of the ANN is given from 9 inputs, 2 hidden layers, and 2 output neurons and it consists of 3 integer numbers that vary in the range $[1,0,-1]$ as it is clear from figures 8 and 11 and based on these 3 values, the node chooses the action to do. The node can move in one of the four directions or remain stable.

The ANN inputs are divided into three sections:

- The first set is an input that shows the number of nodes that are in the same cell.

- The second input set contains four inputs to detect the missing sink connection and to avoid obstacles when one operates in a specific direction.

- The third set subdivision is four inputs that detect overlapping of sensing zone with a neighborhood sensing zone when one operates in a specific direction. 
All inputs listed in each node of the distance measurement were calculated with the other nodes as well as the distance between the node and obstacles:

- The first set of input is useful in determining the number of nodes in the same cell.

- The second set of inputs is a target to make the node spread and maximizes coverage by avoiding overlapping.

- The third one allows the nodes to learn to avoid obstacles.

The signals received by RTL-SDR are enough to record the received and transmitted signals, to monitor problems in sending and receiving between the nodes and to find solutions to these problems.

After collecting the data in a table, it was processed by ANN and the results obtained determined the direction of movement of the node. One of the advantages of the traveling salesman problem (TSP) is to select the best way to visit specific cities. The purpose of using TSP in this research was to make the nodes moving to the specified places have more signal strength between the adjacent nodes and to increase the QoS in terms of coverage and connectivity. The signals received by RTL-SDR and the relative distances in the GA for TSP to feed ANN and make the algorithm work are shown in equation 5 and figure 8 .

$$
a=\operatorname{tansig}(n)=\frac{2}{\left(1+e^{(-2 \times n)}\right)-1}(5)
$$

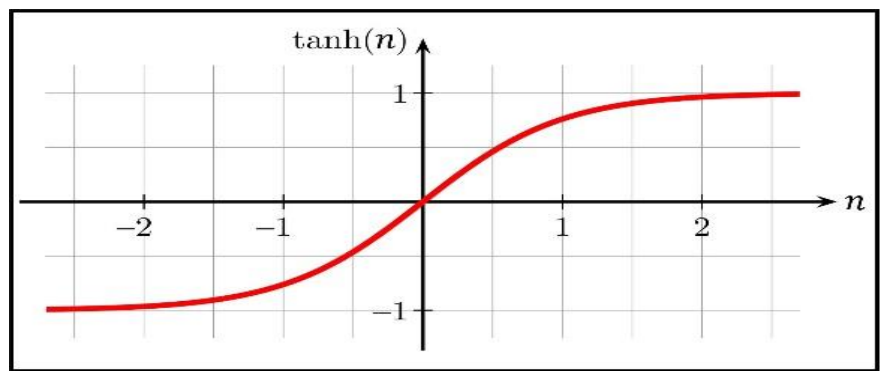

Fig. 8. Tangent-Sigmoid Transfer Function

In order to map the n-dimensional input signal into the m-dimensional output which determines the direction of the movement of the nodes, each neuron uses an activation function and a connection with every other neuron in the network.

$$
\operatorname{Out}_{j}(n)=F\left(\sum i \in N \text { Wij } \cdot \text { out } i(n-1)+b j\right) .
$$

At that time step (n) is the same for all the connections originating in (j). $\mathrm{N}$ is the set of all neurons of ANN and (wij) is the weight of the incoming connection from neuron (i) to neuron (j) while bj is the bias of neuron (j). The activation function (F) is expressed in equation 7. 


$$
F(x)=\left\{\begin{array}{cc}
-1 & \text { if } x \leq-1 \\
0 & \text { if }-1<x<1 \\
1 & \text { if } x \geq 1
\end{array}\right.
$$

The neural network was trained to decide the new coordination of the cell depending on the SDR situation and load on the network service. The set of possible actions is to move in one of the four directions or stay in the current cell.

The whole process is performed in a one-time step for each node of the Wireless Sensor and Robot Networks (WSRN). The training of the ANN consists of performing the described process until a termination condition is satisfied, and shown in figure 9[4].

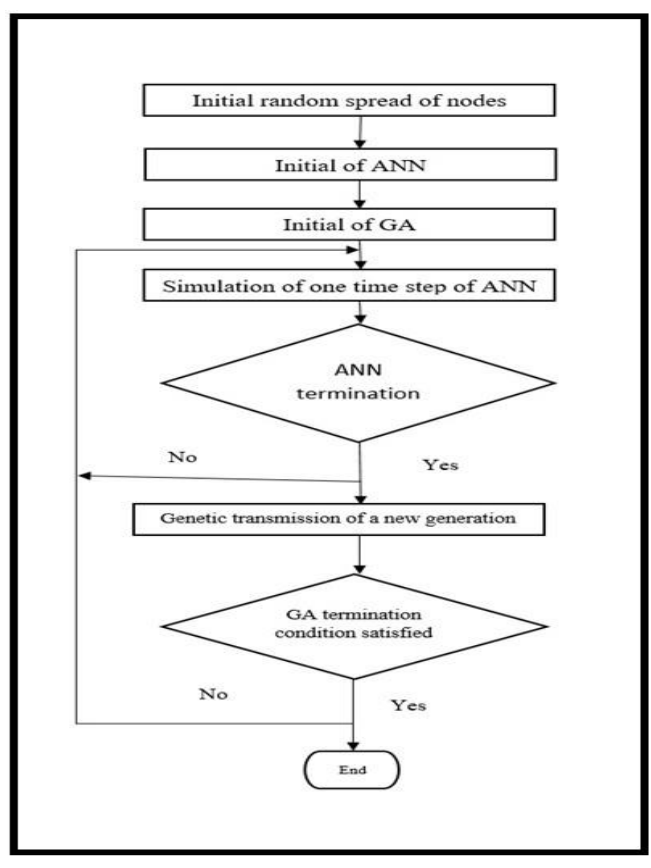

Fig. 9. The training phase of ANN [4]

In every specific area, the node distribution has been identified so that it can cover this area with the minimum number of mobile wireless nodes based on GA for the TSP, where some routes and cities are selected at random, which determines the path of the node to reach the right place. The direction of the movement of the nodes depends on the outputs of the neural obtained from the mobile wireless signal nodes. Each distance between the two cities is a step of the movement of the node and is in the four directions (North - South - East - West). Four secondary directions (north-east, north-west, southeast, and south-west) were included as shown in Figure 10. However, a set of possible movements can be extended to include missing directions in future work. 


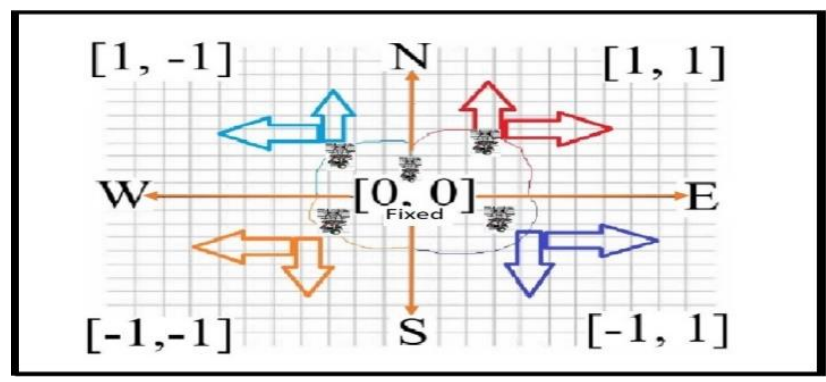

Fig. 10. The possible directions of the mobile node

\subsection{The GA for TSP}

Traditional GA for TSP in this paper is used for training the ANN. The genes linked to the weight of the connections are between each pair of neurons and the bias of each neuron. Different neuronal weights should be selected for future generations through genetic methods (selection, crossover, and mutation). The chromosome chosen for the next generations is the one that best fits[4].

The coverage reaches a maximum depending on the speed of the chromosome. Fitness evaluation has a faster way to calculate the length of the path. It is calculated as a distance matrix. The matrix of distances $(\mathrm{i} 1, \mathrm{i} 2)$ is the distance between node i1 and node i2. To describe shorter tours with higher fitness, the fitness function is selected as $f(x)=1 / d[49]$.

\section{$4 \quad$ Results and Discussion}

\subsection{RTL-SDR results}

SDR was installed and began to read the available signals. The main function of SDR is to discover the RF signals with a specific frequency, which must be determined in advance.

During spectrum analysis, which was achieved by SDR, distortion was detected and removed by the software process on the generated signal. First, the spectrum analyzer for the demodulated signal is recorded and monitored for different central frequencies starting from $(100 \mathrm{MHz})$ to a higher range (see figure 11). The main attenuation signals that are fixed are the harmonic signals that affect the signal quality. Figure 12 shows the spectrum for the demodulated signal with six harmonic signals. 


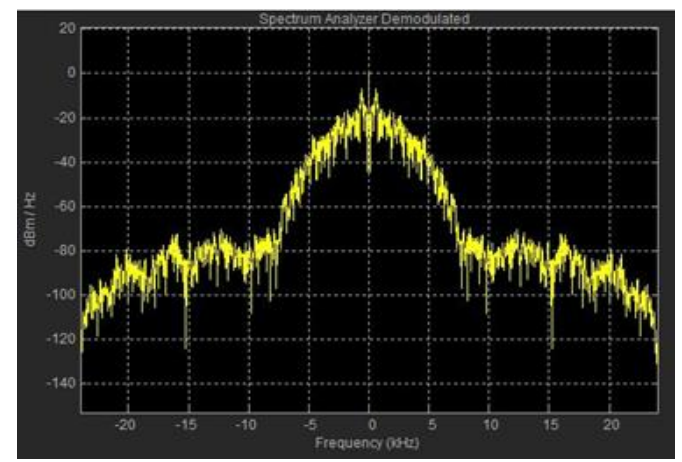

Fig. 11. The Ideal demodulated signal

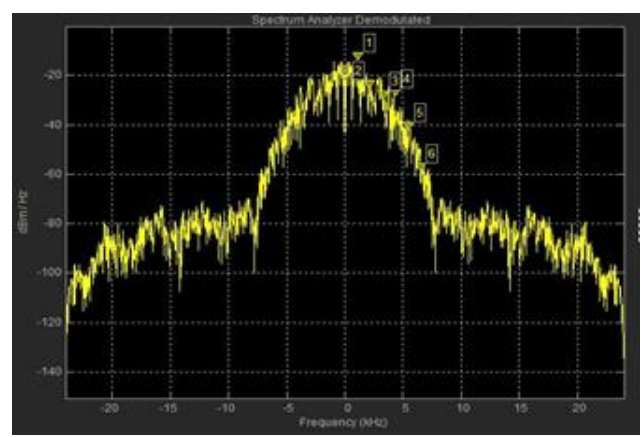

Fig. 12. Power spectrum with 6 harmonics

The effect of attenuation on the main signal is recorded in an additional form by the signal discriminator that compounded all the signals as shown in figure 13.

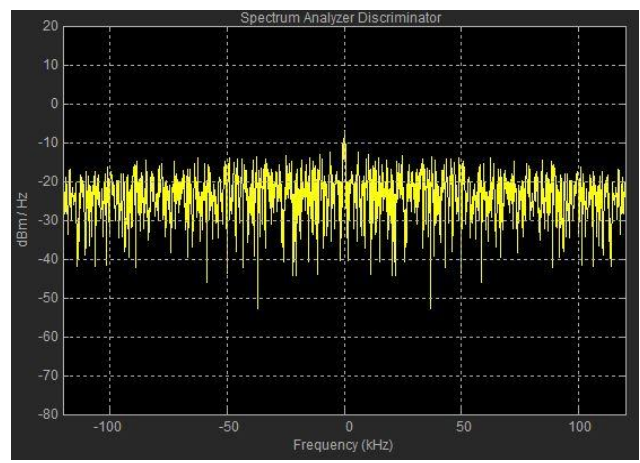

Fig. 13. The spectrum of the discriminator 


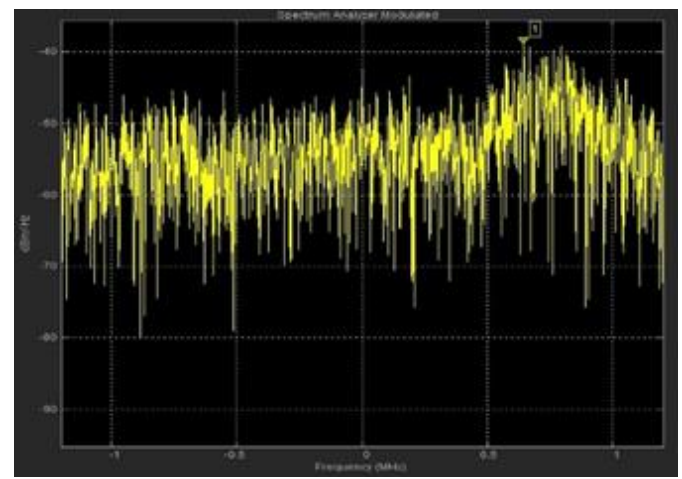

Fig. 14. Spectrum for the modulated signal

Second, the signal will be processed by SDR and all unnecessary components will be filtered to make it ready for the new sender device as shown in figure 14 . There is only one harmonic signal that refers to the main carrier one and there are no unwanted components on the newly generated signal.

The data recorded for the harmonic signals were discovered by (RTL-SDR) receiver to receive signals with a fixed but different central frequency and to demodulate it as shown in figure 14 . The result is recorded in table 1 and it shows the harmonic signal parameters when the fundamental frequency is $(102 \mathrm{MHz})$.

Table 1. RTL-SDR command with (102 MHz) central frequency in the morning.

\begin{tabular}{|c|c|c|}
\hline No. & Harmonic Signal Freq. $\mathbf{~ k H z}$ & P- dB \\
\hline 1 & 0.53 & 14.3 \\
\hline 2 & 1.09 & -10.51 \\
\hline 3 & 1.5 & -8.91 \\
\hline 4 & 2.01 & -15.11 \\
\hline 5 & 2.65 & -22.5 \\
\hline 6 & 3.1 & -13.03 \\
\hline
\end{tabular}




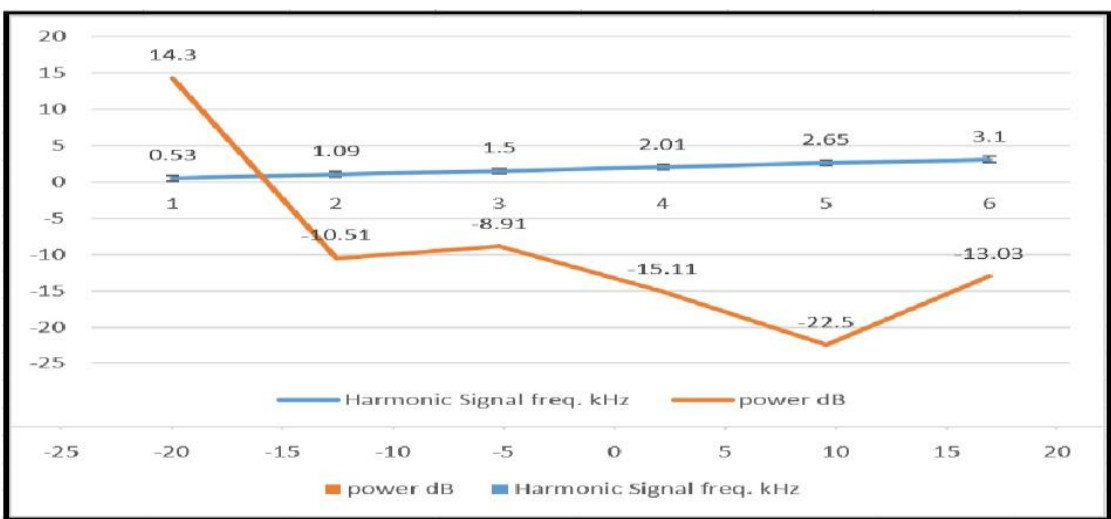

Fig. 15. The power $\mathrm{dB}$ and the harmonic signal for frequency $(102 \mathrm{MHz})$

The Simulink data for the same manner is recorded for the central frequency (486 $\mathrm{MHz}$ ) as shown in Table 2.

Table 2. The harmonic signal parameters for $486 \mathrm{MHz}$ central freq

\begin{tabular}{|c|c|c|}
\hline No. & Harmonic Signal Freq. kHz & Power dB \\
\hline 1 & 20.75 & -47.44 \\
\hline 2 & 41.25 & -6.08 \\
\hline 3 & 62.13 & -9.73 \\
\hline 4 & 83.007 & -7.15 \\
\hline 5 & 0 & 0 \\
\hline 6 & 0 & 0 \\
\hline
\end{tabular}

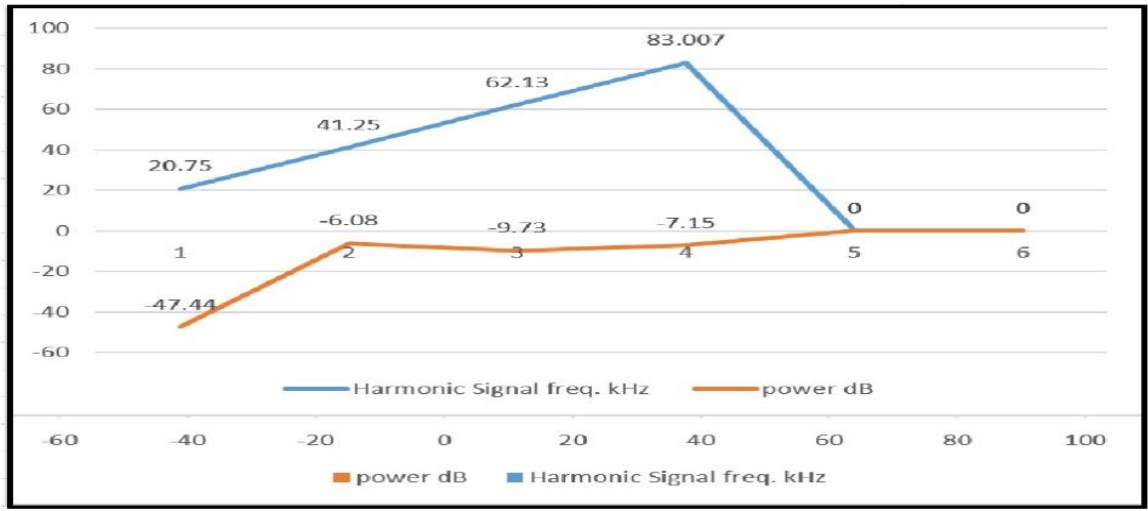

Fig. 16. The harmonic removed for frequency $486 \mathrm{MHz}$

From the last two tables, the central frequency affects two harmonic signals. Therefore, the SDR processes the signal parameters and selects the optimum one depending on the surrounded case. It is one of the SDR obligations to clear the most unwanted components from the signal. 
The last dashes in the table are some removed harmonics from the original signal and do not appear to be ready to send a signal. In the end, as shown in table 2, there is the removal of Harmonics in the signal received after the process of demodulation. The traditional devices were utilized in the processing and the RTL-SDR device was used in this search for processing and to get the target signal. Further, the software was used instead of the hardware. This was one of the objectives of the search.

Table 3. The General affected Parameters on the Received Signal

\begin{tabular}{|c|c|c|c|c|c|}
\hline No & THD & SNR & SINAD & SFDR & F \\
\hline 1 & inf dB & $-18.21 \mathrm{~dB}$ & $-18.77 \mathrm{~dB}$ & $0.28 \mathrm{~dB}$ & $102 \mathrm{Mhz}$ \\
\hline 2 & $-2.22 \mathrm{~dB}$ & $-18.05 \mathrm{~dB}$ & $-17.12 \mathrm{~dB}$ & $0.15 \mathrm{~dB}$ & $102 \mathrm{Mhz}$ \\
\hline 3 & $-4.03 \mathrm{~dB}$ & $-18.55 \mathrm{~dB}$ & $-18.82 \mathrm{~dB}$ & $1.08 \mathrm{~dB}$ & $102 \mathrm{Mhz}$ \\
\hline 4 & $-3.35 \mathrm{~dB}$ & $-18.77 \mathrm{~dB}$ & $-18.87 \mathrm{~dB}$ & $0.09 \mathrm{~dB}$ & $102 \mathrm{Mhz}$ \\
\hline 5 & - inf dB & $-17.45 \mathrm{~dB}$ & $-16.45 \mathrm{~dB}$ & $0.15 \mathrm{~dB}$ & $102 \mathrm{Mhz}$ \\
\hline
\end{tabular}

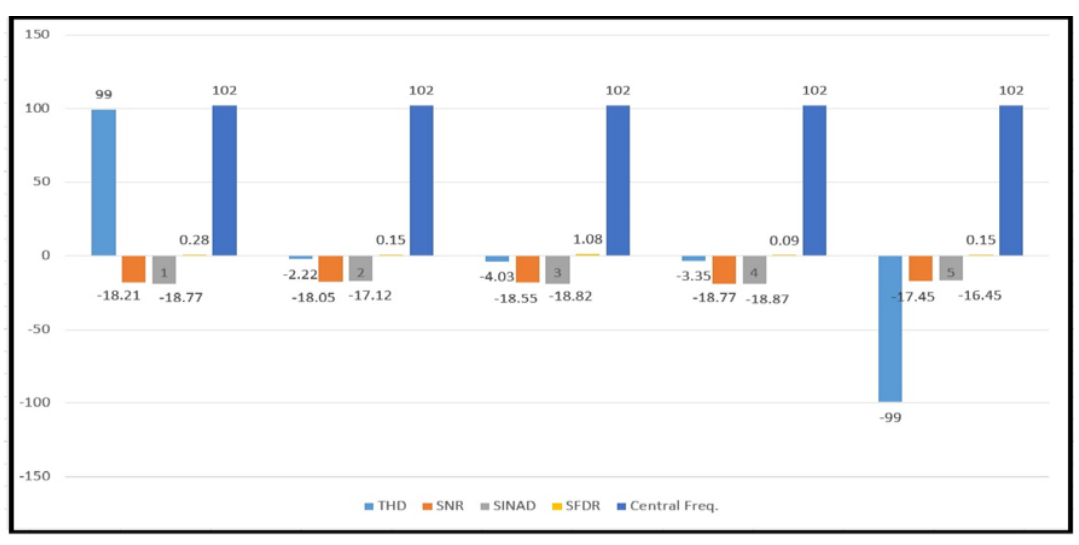

Fig. 17. The general affected parameters on the received signal

Table 4. The general affected parameters on the signal (ready to send)

\begin{tabular}{|c|c|c|c|c|c|}
\hline No & THD & SNR & SINAD & SFDR & F \\
\hline 1 & $2.01 \mathrm{~dB}$ & $-7.21 \mathrm{~dB}$ & $-8.77 \mathrm{~dB}$ & $1.21 \mathrm{~dB}$ & $102 \mathrm{Mhz}$ \\
\hline 2 & $-1.96 \mathrm{~dB}$ & $-8.05 \mathrm{~dB}$ & $-9.12 \mathrm{~dB}$ & $2.45 \mathrm{~dB}$ & $102 \mathrm{Mhz}$ \\
\hline 3 & $14.03 \mathrm{~dB}$ & $-8.55 \mathrm{~dB}$ & $-9.82 \mathrm{~dB}$ & $3.44 \mathrm{~dB}$ & $102 \mathrm{Mhz}$ \\
\hline 4 & $10.59 \mathrm{~dB}$ & $-8.77 \mathrm{~dB}$ & $-8.87 \mathrm{~dB}$ & $0.96 \mathrm{~dB}$ & $102 \mathrm{Mhz}$ \\
\hline 5 & $0.85 \mathrm{~dB}$ & $-6.45 \mathrm{~dB}$ & $-6.45 \mathrm{~dB}$ & $0.05 \mathrm{~dB}$ & $102 \mathrm{Mhz}$ \\
\hline
\end{tabular}




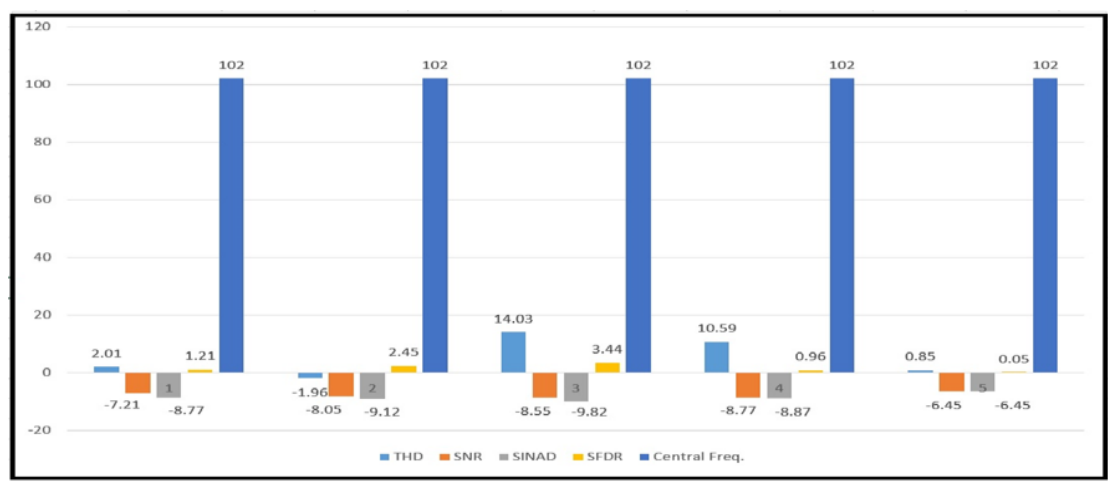

Fig. 18. The general affected parameters on the signal demodulated by RTL-SDR

Finally, as shown in Tables 3 and 4 and Figures 17 and 18 where the harmonic is removed in the received signal after the demodulation process with the focus on signal processing using SDR, there is a significant difference in signal measurements recorded in the last two tables. The signal received contains more distortion and unwanted components compared to the signal that is demodulated by the RTL-SDR, therefore it is possible to compare the two results in the tables from THD, SNR, SINAD, and SFDR where a typical signal is obtained for the purity. It was accomplished by the SDR technique.

Table 5 shows some cases recorded for the affected general parameters with RTLSDR signals analyzed by ANN. Based on this, the results in the table below show the direction of the movement of the node.

Table 5. The output of ANN for selecting the direction of nodes

\begin{tabular}{|c|c|c|c|c|c|}
\hline No & THD & SNR & SINAD & SFDR & F \\
\hline 1 & inf dB & $-18.21 \mathrm{~dB}$ & $-18.77 \mathrm{~dB}$ & $0.28 \mathrm{~dB}$ & $102 \mathrm{Mhz}$ \\
\hline 2 & $-2.22 \mathrm{~dB}$ & $-18.05 \mathrm{~dB}$ & $-17.12 \mathrm{~dB}$ & $0.15 \mathrm{~dB}$ & $102 \mathrm{Mhz}$ \\
\hline 3 & $-4.03 \mathrm{~dB}$ & $-18.55 \mathrm{~dB}$ & $-18.82 \mathrm{~dB}$ & $1.08 \mathrm{~dB}$ & $102 \mathrm{Mhz}$ \\
\hline 4 & $-3.35 \mathrm{~dB}$ & $-18.77 \mathrm{~dB}$ & $-18.87 \mathrm{~dB}$ & $0.09 \mathrm{~dB}$ & $102 \mathrm{Mhz}$ \\
\hline 5 & - inf dB & $-17.45 \mathrm{~dB}$ & $-16.45 \mathrm{~dB}$ & $0.15 \mathrm{~dB}$ & $102 \mathrm{Mhz}$ \\
\hline
\end{tabular}

As shown in Table 7, the results obtained by RTL-SDR were analyzed by ANN. When the result is $(0,0)$ it means that the node remains fixed depending on the signal received. If the result is $(1,1)$, it means that the movement is going to north-east and if the result is $(1,-1)$ it indicates that the action is in the north-west direction. For a result indicating $(-1,1)$ the movement is towards the south-east and if the result is $(-1,-1)$ it indicates that the action is south-west. After determining the direction of the movement by ANN, the role of the GA for the TSP is to begin to select the shortest way to go to the specified position, and this is the objective of the movement in this research[50]. 


\subsection{GA for TSP results}

The routes proposed by the GA to the 3 salesmen were fixed as shown in figure 19 . The figure shows the links and location to pass through and return to the starting point. Figure 20 shows the number of iterations and more details for route finding.
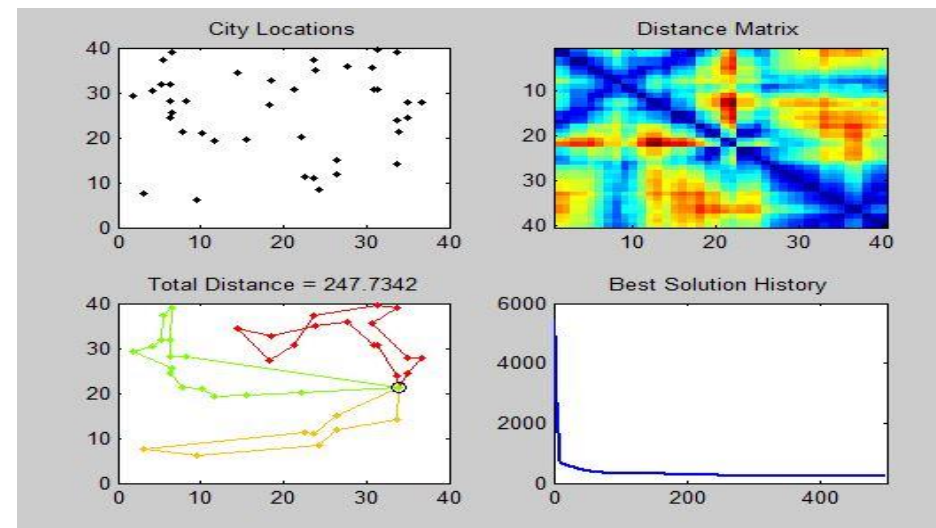

Fig. 19. The route finding and sales point determination using ANN and GA for STP

The four parts in the above figure entail; one, the 40 cities which are the locations that TSP must-visit, two, the distance matrix that contains the specific kilometers between any two nodes locations and is displayed by colors each of them representing a particular value of distance. The blue color is the nearest one, and the yellow is the furthest location. Three, the total distance is the total distance which the salesman passes through from the starting point until the end of all roads. Finally, the best solution shows the total distance in kilometer required to be taken to visit all mobile nodes position given by the paths, which was selected paths randomly in this area starting from (0 to 6000) paths, and these paths are selected by the TSP to visit the nearest city.

As shown in Figure 20, three nodes were selected and each of them moved in a specific direction depending on the output of the AI. As each node visited a respective position specified in the same direction it leads to the coverage of the area identified using three nodes.

Another tries to find the optimum routes for the salesman and sales location fixed as shown in figure 20. It shows a different map and the new distribution for the locations where the specific area covered has fewer distances. 

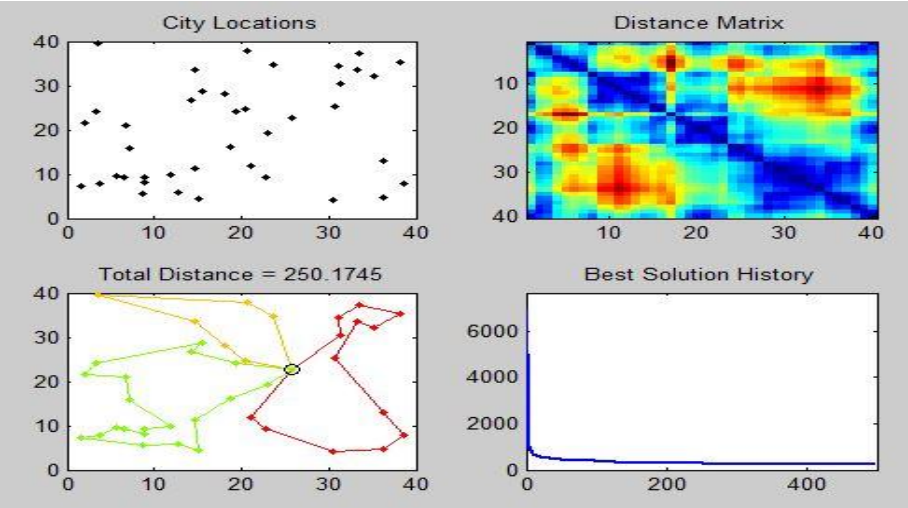

Fig. 20. Another solution for the mobile node route determination

The figures above show how the three salesmen cover the whole given region and the transition between them to produce optimum service. Figures 19 and 20 give more details about the routes and locations determination. In these two figures, the effect of selecting three nodes on the coverage is shown. The movement of each node in a specific direction increases coverage in the specified area but increases the number of nodes leading to an increase in the cost and the length of the path. This distribution is based on the decision of ANN and the genetic optimization for TSP to select the direction of movement and distance.

\subsection{The ANN and GA for MTSP effects on coverage}

First, nodes location refers to the proposed coordinates for the service nodes that receive and transmit the user information after fixed nodes on these points. Secondly, the routes matrix refers to the link the nodes communicate with and it represents paths. The total distance is the complete distance from the starting point for node location until it reaches the last position for node location. Finally, the best solution translates the learning response for the system and how to reach the best node distribution with the smallest link distance.

In this paper, the interactive combination of controlled mobility for nodes and SDRs in a fully distributed fashion allowed a high degree of self-configuration that increased the QoS in terms of coverage and connectivity, and the nodes randomly distributed in a specific area.

This research focused on the performance of the best service and lowest cost because of the mobility capabilities of the nodes and SDR flexibility. The number of nodes was reduced in the same area respectively. As shown in figures 21 the 40, nodes were chosen but they show that the coverage is high and expensive because of the increase in the number of nodes. When the number of nodes was reduced to 20 nodes as shown in figure 22 in the same area and the coverage was also high, the number of nodes became significant. The continuous reduction of the number of nodes went to 10 nodes as shown in figure 23 and the follow-up result was high coverage. 
Finally, when the nodes were reduced to 4 as shown in figure 24 the coverage was high at the same time. This service was due to the mobility of the nodes, according to the algorithm specified in this paper, which moves towards the appropriate place to increase the coverage and connectivity in the specific area and perform the best service. This was the research objective, and it belongs to the capabilities of nodes mobility together with the SDR and AI capabilities in a synergic way to manage the system see Figures.

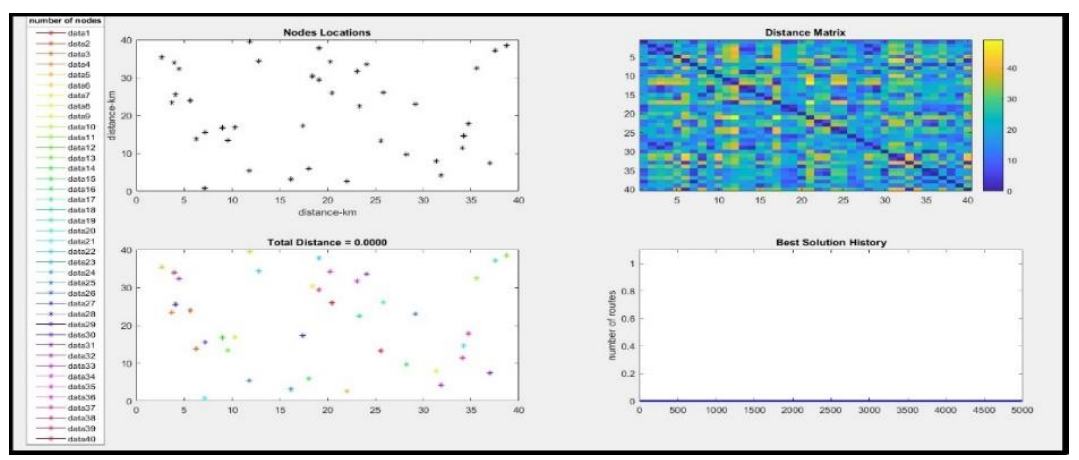

Fig. 21. Node determination results using AI and GA for TSP when $n=40$

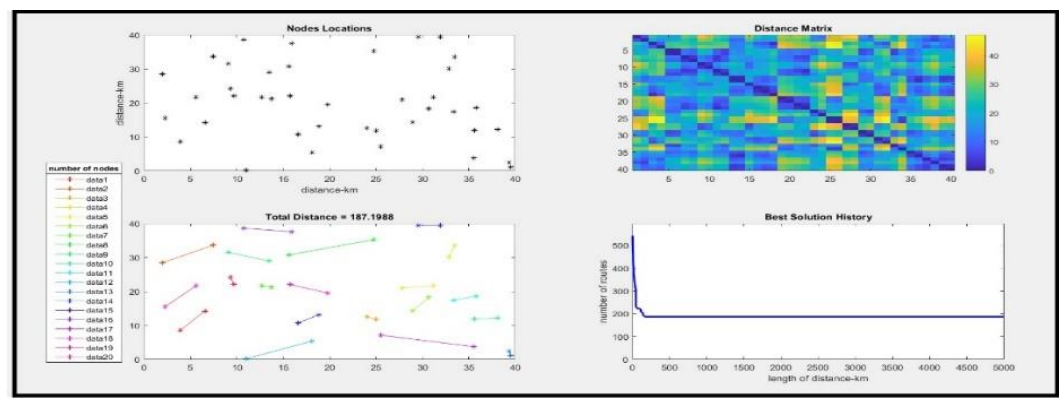

Fig. 22. Node determination results when $n=20$

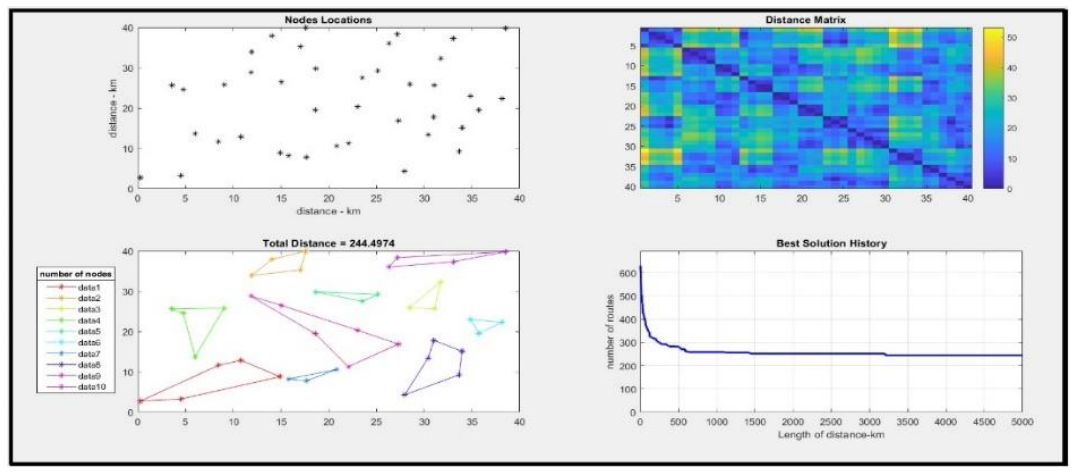

Fig. 23. Node determination results when $n=10$ 


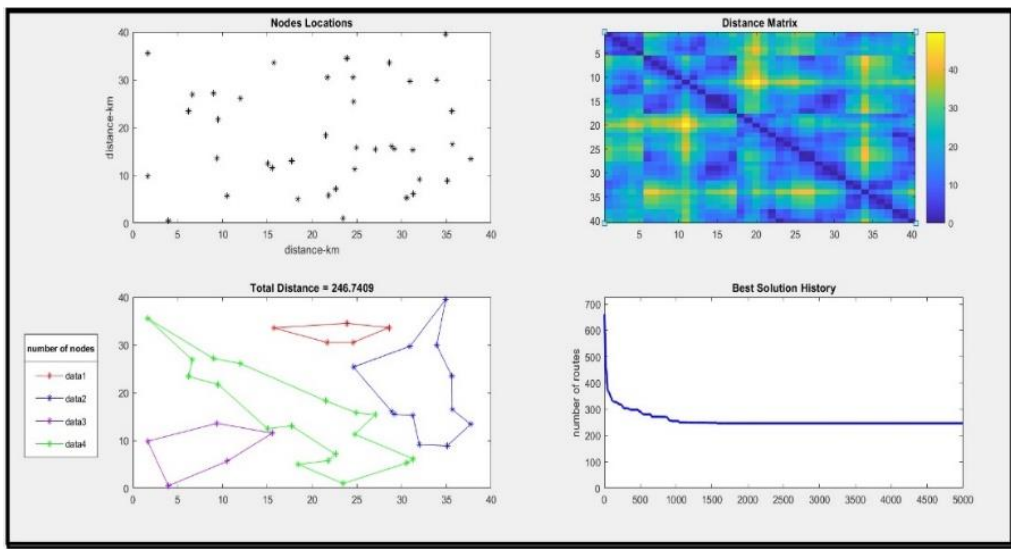

Fig. 24. Node determination results when $n=4$

\section{Conclusion}

In this paper, the distribution capabilities of both ANN and GA for TSP has been proposed to select the most suitable positioning for the mobile nodes equipped with SDR capabilities. The node studied in this work could move towards new locations by applying the concept of controlled mobility by using only local information. The interactive combination of controlled mobility and SDRs in a fully distributed fashion allowed a high degree of self-configuration that increased the QoS in terms of coverage and connectivity. The combination of SDR and ANN allowed improvement in the management of wireless communication systems in terms of flexibility and economic viability.

\section{$6 \quad$ References}

[1] D. A. Zebari, H. Haron, S. R. Zeebaree, and D. Q. Zeebaree, "Multi-Level of DNA Encryption Technique Based on DNA Arithmetic and Biological Operations," in 2018 International Conference on Advanced Science and Engineering (ICOASE), 2018, pp. 312-317: IEEE. https://doi.org/10.1109/icoase.2018.8548824

[2] M. Gandetto, M. Guainazzo, and C. S. Regazzoni, "Use of time-frequency analysis and neural networks for mode identification in a wireless software-defined radio approach," EURASIP Journal on Advances in Signal Processing, vol. 2004, no. 12, p. 863653, 2004. https://doi.org/10.1155/s1110865704407057

[3] F. Guerriero, V. Loscrí, P. Pace, and R. Surace, "Neural networks and SDR modulation schemes for wireless mobile nodes: A synergic approach," Ad Hoc Networks, vol. 54, pp. 17-29, 2017. https://doi.org/10.1016/j.adhoc.2016.09.016

[4] V. Loscrí, P. Pace, and R. Surace, "Multi-objective evolving neural network sup-porting SDR modulations management," in 2013 IEEE 24th Annual International Symposium on Personal, Indoor, and Mobile Radio Communications (PIMRC), 2013, pp. 1946-1951: IEEE. https://doi.org/10.1109/pimrc.2013.6666462 
[5] H. A. AL-Behadili, M. N. S. Almaliki, and S. K. A. AlWane, "A Ray Tracing Model for Wireless Communications," International Journal of Interactive Mobile Technologies (iJIM), vol. 13, no. 11, pp. 245-251, 2019. https://doi.org/10.3991/ijim.v13i11.11730

[6] J. Jagannath et al., "Artificial neural network based automatic modulation classification over a software defined radio testbed," in 2018 IEEE International Conference on Communications (ICC), 2018, pp. 1-6: IEEE. https://doi.org/10.1109/icc.2018.8422346

[7] X. Zhou, M. Sun, G. Y. Li, and B.-H. F. Juang, "Intelligent wireless communications enabled by cognitive radio and machine learning," China Communications, vol. 15, no. 12, pp. 16-48, 2018.

[8] A. Dutta, D. Saha, D. Grunwald, and D. Sicker, "An architecture for software defined cognitive radio," in 2010 ACM/IEEE Symposium on Architectures for Net-working and Communications Systems (ANCS), 2010, pp. 1-12: IEEE. https://doi.org/10.1145/1872 $\underline{007.1872014}$

[9] S. Kaur and V. Bahl, "Software Defined Radio Technology-Next Generation Intelligent Radios," International Journal of Engineering Sciences \& Research Technology, vol. 4, p. 12, 2015.

[10] T. Ulversoy, "Software defined radio: Challenges and opportunities," IEEE Communications Surveys \& Tutorials, vol. 12, no. 4, pp. 531-550, 2010. https://doi.org/10.1109/surv. 2010.032910.00019

[11] I. F. Akyildiz, P. Wang, and S.-C. Lin, "SoftAir: A software defined networking architecture for 5G wireless systems," Computer Networks, vol. 85, pp. 1-18, 2015. https://doi.org/ 10.1016/j.comnet.2015.05.007

[12] G. Aloi et al., "STEM-Net: an evolutionary network architecture for smart and sustainable cities," Transactions on Emerging Telecommunications Technologies, vol. 25, no. 1, pp. 2140, 2014. https://doi.org/10.1002/ett.2785

[13] I. F. Akyildiz, W.-Y. Lee, M. C. Vuran, and S. Mohanty, "NeXt generation/dynamic spectrum access/cognitive radio wireless networks: A survey," Computer networks, vol. 50, no. 13, pp. 2127-2159, 2006. https://doi.org/10.1016/j.comnet.2006.05.001

[14] C. Blümm, C. Heller, and R. Weigel, "SDR OFDM waveform design for a UGV/UAV communication scenario," Journal of Signal Processing Systems, vol. 69, no. 1, pp. 11-21, 2012. https://doi.org/10.1007/s11265-011-0640-8

[15] I. F. Akyildiz, A. Lee, P. Wang, M. Luo, and W. Chou, "Research challenges for traffic engineering in software defined networks," IEEE Network, vol. 30, no. 3, pp. 52-58, 2016. https://doi.org/10.1109/mnet.2016.7474344

[16] B. B. Godbole and D. S. Aldar, "Performance improvement by changing modulation methods for software defined radios," arXiv preprint arXiv:1212.0114, 2012.

[17] G. Aloi et al., "Software defined radar: synchronization issues and practical implementation," in Proceedings of the 4th International Conference on Cognitive Radio and Advanced Spectrum Management, 2011, pp. 1-5.

[18] J. Van der Merwe, J. Malan, F. Maasdorp, and W. Du Plessis, "Multi-channel soft-ware defined radio experimental evaluation and analysis," Proc. Instituto Tecnologico de Aeronautica (ITA), 2014.

[19] H. Lee et al., "Software defined radio-a high performance embedded challenge," in International Conference on High-Performance Embedded Architectures and Compilers, 2005, pp. 6-26: Springer. https://doi.org/10.1007/11587514_3

[20] H. Lee, "A baseband processor for software defined radio terminals," University of Michigan, Ann Arbor Ph. D Dissertation, 2007.

[21] R. Chávez-Santiago, A. Mateska, K. Chomu, L. Gavrilovska, and I. Balasingham, "Applications of software-defined radio (SDR) technology in hospital environments," in 2013 35th 
Annual International Conference of the IEEE Engineering in Medicine and Biology Society (EMBC), 2013, pp. 1266-1269: IEEE. https://doi.org/10.1109/embc.2013.6609738

[22] T. TAKEUCHI, A. HONDA, H. WATANABE, Y. ETO, Y. FUJITA, and M. YAGI, "Emergency Mobile Radio Network based on Software-Defined Radio."

[23] M. Dillinger, K. Madani, and N. Alonistioti, Software defined radio: Architectures, systems and functions. John Wiley \& Sons, 2005.

[24] S. Salah and R. Zaghal, "DSDV Extension to Enhance the Performance of Ad Hoc Networks in High Diverse-Velocity Environments," International Journal of Inter-active Mobile Technologies (iJIM), vol. 14, no. 06, pp. 95-114, 2020. https://doi.org/10.3991/ijim. $\underline{\mathrm{v} 14 \mathrm{i} 06.11889}$

[25] M. Dardaillon, K. Marquet, T. Risset, J. Martin, and H.-P. Charles, "A new compilation flow for software-defined radio applications on heterogeneous MPSoCs," ACM Transactions on Architecture and Code Optimization (TACO), vol. 13, no. 2, pp. 1-25, 2016. https://doi.org/10.1145/2910583

[26] D. Sinha, A. K. Verma, and S. Kumar, "Software defined radio: Operation, challenges and possible solutions," in 2016 10th International Conference on Intelligent Systems and Control (ISCO), 2016, pp. 1-5: IEEE. https://doi.org/10.1109/isco.2016.7727079

[27] M. Mukesh, L. Abhishek, and R. Bhambare, "QPSK modulator and demodulator using FPGA for SDR," International. Journal of Engineering Research and Applications, vol. 4, no. 4, pp. 394-397, 2014.

[28] A. M. Abdulazeez and A. S. Tahir, "Design and Implementation of Advanced Encryption Standard Security Algorithm using FPGA," Int. J. of Computers \& Technology, vol. 4, no. 9, pp. 1988-1993, 2013.

[29] A. Kamaleldin et al., "A reconfigurable hardware platform implementation for software defined radio using dynamic partial reconfiguration on Xilinx Zynq FPGA," in 2017 IEEE 60th International Midwest Symposium on Circuits and Systems (MWSCAS), 2017, pp. 1540-1543: IEEE. https://doi.org/10.1109/mwscas.2017.8053229

[30] C. Sacchi and R. Passerone, "A new vision of software defined radio: from academic experimentation to industrial explotation," 2011.

[31] S. Bassam et al., "A generic architecture for smart multi-standard software defined radio systems," in SDR, 2009, vol. 9, pp. 1-4.

[32] O. Popescu, S. Abraham, and S. El-Tawab, "A mobile platform using software defined radios for wireless communication systems experimentation," 2017. https://doi.org/10.182 $\underline{60 / 1-2--27482}$

[33] F. A. de Figueiredo, X. Jiao, W. Liu, and I. Moerman, "Radio hardware virtualization for software-defined wireless networks," Wireless Personal Communications, vol. 100, no. 1, pp. 113-126, 2018. https://doi.org/10.1007/s11277-018-5619-3

[34] F. Xu, H. Yao, C. Zhao, and C. Qiu, "Towards next generation software-defined radio access network-architecture, deployment, and use case," EURASIP Journal on Wireless Communications and Networking, vol. 2016, no. 1, p. 264, 2016. https://doi.org/10.1186/s13638$\underline{016-0762-6}$

[35] T. Padmapriya and S. Manikanthan, "Security and Routing Protocol for 5G Wire-less Mobile Networks," International Journal of Interactive Mobile Technologies (iJIM), vol. 14, no. 05, pp. 164-175, 2020. https://doi.org/10.3991/ijim.v14i05.13363

[36] S. Sun, M. Kadoch, L. Gong, and B. Rong, "Integrating network function virtualization with SDR and SDN for 4G/5G networks," IEEE Network, vol. 29, no. 3, pp. 54-59, 2015. https://doi.org/10.1109/mnet.2015.7113226

[37] [39] D. F. Macedo, D. Guedes, L. F. Vieira, M. A. Vieira, and M. Nogueira, "Programmable networks-From software-defined radio to software-defined networking," IEEE 
communications surveys \& tutorials, vol. 17, no. 2, pp. 1102-1125, 2015. https://doi.org/ $\underline{10.1109 / \mathrm{comst} .2015 .2402617}$

[38] H. Yoon, J. Um, J.-S. Park, and B.-J. Jang, "SDR-based frequency interference emulator in the space-time domain and its application," Journal of electromagnetic engineering and science, vol. 18, no. 1, pp. 58-62, 2018. https://doi.org/10.26866/jees.2018.18.1.58

[39] R. K. Miranda et al., "Implementation of Improved Software Defined Radio Modulation Scheme and Command and Telemetry Software Interface for Small Satellites in 5G Systems," in ICOF 2016; 19th International Conference on OFDM and Frequency Domain Techniques, 2016, pp. 1-7: VDE.

[40] G. Piccinini, "The First computational theory of mind and brain: a close look at mcculloch and pitts's "logical calculus of ideas immanent in nervous activity"," Synthese, vol. 141, no. 2, pp. 175-215, 2004. https://doi.org/10.1023/b:synt.0000043018.52445.3e

[41] T. H. Abraham, "(Physio) logical circuits: The intellectual origins of the McCul-loch-Pitts neural networks," Journal of the History of the Behavioral Sciences, vol. 38, no. 1, pp. 3-25, 2002. https://doi.org/10.1002/jhbs. 1094

[42] A. S. Eesa, Z. Orman, and A. M. A. Brifcani, "A novel feature-selection approach based on the cuttlefish optimization algorithm for intrusion detection systems," Expert Systems with Applications, vol. 42, no. 5, pp. 2670-2679, 2015. https://doi.org/10.1016/j.eswa.2014.11. $\underline{009}$

[43] A. Sharma and A. Chopra, "Artificial neural networks: Applications in management," Journal of Business and Management, vol. 12, no. 5, pp. 32-40, 2013.

[44] W. Samek, S. Stanczak, and T. Wiegand, "The convergence of machine learning and communications," arXiv preprint arXiv:1708.08299, 2017.

[45] D. Q. Zeebaree, H. Haron, and A. M. Abdulazeez, "Gene selection and classification of microarray data using convolutional neural network," in 2018 International Conference on Advanced Science and Engineering (ICOASE), 2018, pp. 145-150: IEEE. https://doi.org/ 10.1109/icoase.2018.8548836

[46] W. Kester, "Mt-001: Taking the mystery out of the infamous formula," snr= $6.02 n+1.76$ db," and why you should care," REV. 0, pp. 10-03, 2005.

[47] Z. H. Ahmed, "Genetic algorithm for the traveling salesman problem using sequential constructive crossover operator," International Journal of Biometrics \& Bioinformatics (IJBB), vol. 3, no. 6, p. 96, 2010.

[48] D. Q. Zeebaree, H. Haron, A. M. Abdulazeez, and S. R. Zeebaree, "Combination of K-means clustering with Genetic Algorithm: A review," International Journal of Applied Engineering Research, vol. 12, no. 24, pp. 14238-14245, 2017.

\section{$7 \quad$ Authors}

Faiq A. Mohammed Bargarai He holds a B. Eng. degree in the University of Hadb'a in the Department of Computer technology engineering, and I worked at the Bardarash Bank of the Department of Information Technology and worked at Taren Net Communications Company and then I started studying for a master's degree in Turkey at the University of Siirt and research specialized in software-defined radio defined, and currently, I work manager for registration department and teaching at the Bardarash Institute Duhok Polytechnic University.

Adnan Mohsin Abdulazeez President of Duhok Polytechnic University, Professor of Computer Engineering and Science. Dr. Abdulazeez holds Ph.D. in Computer 
Engineering, M.Sc. in Computer and Control Engineering, and his B.Sc. in Electrical and Electronic Engineering. He was the Dean of Duhok Technical Institute until August 2012. He has been assigned as head of many scientific departments and committees in public and private universities in Kurdistan Region and Iraq. He has published a number of articles and researches in local and international scientific journals most of them focused on intelligence systems. In addition, he supervised more than thirty Ph.D. and M.Sc. Students.

Volkan Müjdat Tiryaki Dr. Tiryaki is currently an Assistant Professor in the Department of Computer Engineering at Siirt University. He received his B.S. and M.S. degrees in Electronics and Industrial Engineering from Istanbul University. He earned his $\mathrm{PhD}$ from the Department of Electrical and Computer Engineering at Michigan State University in 2013. He has authored five international and a national journal article. His research interests include computer aided detection and diagnosis of breast cancer, semantic segmentation, nanoscale cues for regenerative neural cell systems, and quantitative biomaterials characterization.

Diyar Qader Zeebaree holds Ph.D. in computer science at UTM in Malaysia, M.Sc. in computer information systems, and his B.Sc. in computer science. Currently he is working in Duhok Polytechnic University as director of research center. He has published a number of articles and researches in international scientific journals most of them focused on Artificial Intelligence, Machine Learning, Deep Learning, image processing, security and Medical images.

Article submitted 2020-03-12. Resubmitted 2020-04-18. Final acceptance 2020-04-20. Final version published as submitted by the authors. 